\title{
INternational Study Group of Pediatric Pancreatitis: In Search for a CuRE (INSPPIRE 2) Cohort Study: Design and Rationale From the Consortium for the Study of Chronic Pancreatitis, Diabetes, and Pancreatic Cancer
}

\author{
Aliye Uc, MD*, Emily R. Perito, MD ${ }^{\dagger}$, John F. Pohl, MD $\ddagger$, Uzma Shah, MBBS§, Maisam Abu- \\ El-Haija, MD", Bradley Barth, MDף, Melena D. Bellin, MD\#, Kate M. Ellery, DO ${ }^{\star *}$, Douglas S. \\ Fishman, MD ${ }^{\dagger \dagger}$, Cheryl E. Gariepy, MD ${ }^{\ddagger \ddagger}$, Matthew J. Giefer, MD§§, Tanja Gonska, MD ${ }^{\prime \prime \prime \prime}$, \\ Melvin B. Heyman, MD, MPH ${ }^{\dagger}$, Ryan W. Himes, MD ${ }^{\dagger \dagger}$, Sohail Z. Husain, MD ${ }^{* \star}$, Asim \\ Maqbool, MD ${ }^{\text {II }}$, Maria R. Mascarenhas, MD IT, Brian A. McFerron, MD\#\#, Veronique D. \\ Morinville, MD ${ }^{\star \star \star}$, Tom K. Lin, MD", Quin Y. Liu, MD ${ }^{\dagger \dagger}$, Jaimie D. Nathan, MD", Sue J. Rhee, \\ $M^{\dagger}{ }^{\dagger}$, Chee Y. Ooi, MBBS, PhD $¥ \neq \ddagger$, Zachary M. Sellers, MD, PhD§§§, Sarah Jane \\ Schwarzenberg, MD\#, Jose Serrano, MD, PhD"llll, David M. Troendle, MDף, Steven L. Werlin, \\ MDITI, Michael Wilschanski, MBBS \#\#\#, Yuhua Zheng, MS MD ${ }^{* \star *}$, Ying Yuan, PhD ${ }^{\dagger \dagger}{ }^{\dagger}$, Mark \\ E. Lowe, MD, PhD $\ddagger \ddagger \neq$, and on behalf of the Consortium for the Study of Chronic \\ Pancreatitis, Diabetes, and Pancreatic Cancer (CPDPC) \\ "Stead Family Department of Pediatrics, University of lowa, Stead Family Children's Hospital, \\ lowa City, IA \\ tDepartment of Pediatrics, University of California San Francisco, San Francisco, CA \\ ‡Department of Pediatrics, University of Utah, Salt Lake City, UT \\ $\S$ Department of Pediatrics, Massachusetts General Hospital for Children, Harvard Medical School, \\ Boston, MA \\ "Department of Pediatrics, Cincinnati Children's Hospital Medical Center, Cincinnati, OH \\ IDepartment of Pediatrics, University of Texas Southwestern Medical School, Dallas, TX \\ \#Department of Pediatrics, University of Minnesota Masonic Children's Hospital, Minneapolis, MN \\ ${ }^{* *}$ Department of Pediatrics, Children's Hospital of Pittsburgh, Pittsburgh, PA \\ t†Department of Pediatrics, Baylor College of Medicine, Houston, TX \\ 抽epartment of Pediatrics, Nationwide Children’s Hospital, Columbus, $\mathrm{OH}$
}

\footnotetext{
Address correspondence to: Aliye Uc, MD, Stead Family Department of Pediatrics, University of Iowa Health Care, 200 Hawkins Drive, BT 1120-C, Iowa City, IA 52242 ( aliye-uc@uiowa.edu). Phone: 319-356-2950.

Conflicts: Dr. Mark Lowe is on the Board of Directors of the National Pancreas Foundation; receives royalties from Millipore Inc and UpToDate. Drs. Tanja Gonska and Michael Wilschanski received a research grant from Vertex Pharmaceuticals. Dr. Sohail Husain owns equity in PrevCon. Dr. John Pohl is on the speaker's bureau for Medical Education Resources, Inc.; Dr. Melena Bellin is a consultant for AbbVie Inc and ARIEL Precision Medicine. Dr. Aliye Uc is a member of American Board of Pediatrics, Subboard of Pediatric Gastroenterology. The other authors declare no conflicts of interest.

All authors are responsible for reported research. They have participated in the concept and design; collection, analysis and interpretation of data; writing and revising of the manuscript, and decision to submit the manuscript for publication.
} 
\$Department of Pediatrics, Seattle Children's Hospital, Seattle, WA

III'Department of Pediatrics, Hospital for Sick Children, Toronto, Canada

†Department of Pediatrics, University of California San Francisco, San Francisco, CA

${ }^{+\dagger}$ Department of Pediatrics, Baylor College of Medicine, Houston, TX

${ }^{* *}$ Department of Pediatrics, Children's Hospital of Pittsburgh, Pittsburgh, PA

ITDepartment of Pediatrics, Children's Hospital of Philadelphia, Philadelphia, PA

TIDepartment of Pediatrics, Children's Hospital of Philadelphia, Philadelphia, PA

\#\#Department of Pediatrics, Riley Hospital for Children, Indiana University School of Medicine, Indianapolis, IN

${ }^{\star \star *}$ Department of Pediatrics, Montreal Children's Hospital, McGill University, Montreal, Canada

"Department of Pediatrics, Cincinnati Children's Hospital Medical Center, Cincinnati, $\mathrm{OH}$

t+†Department of Pediatrics and Medicine, Cedars-Sinai Medical Center, Los Angeles, CA

"Division of Pediatric General and Thoracic Surgery, Cincinnati Children's Hospital Medical

Center, Cincinnati, $\mathrm{OH}$

†Department of Pediatrics, University of California San Francisco, San Francisco, CA

¥¥Department of Pediatrics, School of Women's and Children's Health, Medicine, University of New South Wales and Sydney Children's Hospital, Sydney, Australia

$\S \S \S$ Department of Pediatrics, Stanford University, Stanford, CA

\#Department of Pediatrics, University of Minnesota Masonic Children's Hospital, Minneapolis, MN

IIII'Division of Digestive Diseases and Nutrition, National Institute of Diabetes and Digestive and Kidney Diseases (NIDDK), Bethesda, MD

IDepartment of Pediatrics, University of Texas Southwestern Medical School, Dallas, TX

TथाDepartment of Pediatrics, Medical College of Wisconsin, Milwaukee, WI

\#\#\#Department of Pediatrics, Hadassah Hebrew University Hospital, Jerusalem, Israel

${ }^{\star \star * \star}$ Department of Pediatrics, Children's Hospital Los Angeles, Los Angeles, CA

ttttDepartment of Biostatistics, The University of Texas, MD Anderson Cancer Center, Houston, TX

$\ddagger \neq \ddagger \neq$ Department of Pediatrics, Washington University School of Medicine, St. Louis, MO

\section{Abstract}

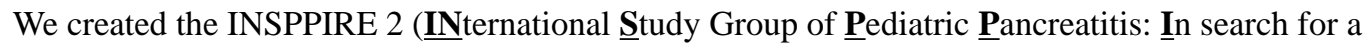
cuRE) Cohort to study the risk factors, natural history and outcomes of pediatric acute recurrent pancreatitis (ARP) and chronic pancreatitis (CP). Patient and physician questionnaires collect information on demographics, clinical history, family and social history, and disease outcomes. Health-related quality of life, depression, and anxiety are measured with validated questionnaires.

Pancreas. Author manuscript; available in PMC 2019 November 01. 
Information entered on paper questionnaires is transferred into a database managed by Consortium for the Study of Chronic Pancreatitis, Diabetes and Pancreatic Cancer's (CPDPC) Coordinating and Data Management Center (CDMC). Biosamples are collected for DNA isolation and analysis of most common pancreatitis-associated genes.

Twenty-two sites (18 in United States, 2 in Canada, and 1 each in Israel and Australia) are participating in the INSPPIRE 2 study. These sites have enrolled 211 subjects into the INSPPIRE 2 database toward our goal to recruit over 800 patients in 2 years. The INSPPIRE 2 Cohort Study is an extension of the INSPPIRE Cohort Study with a larger and more diverse patient population. Our goals have expanded to include evaluating risk factors for $\mathrm{CP}$, its sequelae, and psychosocial factors associated with pediatric ARP and CP.

\section{Keywords}

children; registry; pancreatitis

\section{INTRODUCTION}

Once considered an uncommon disease, the incidence of pediatric acute pancreatitis (AP) has increased over the last $10-20$ years, affecting approximately 1 in 10,000 children. ${ }^{1-7}$ Acute recurrent pancreatitis (ARP), characterized as two or more discrete episodes of acute pancreatitis, is reported in $15-35 \%$ of children following an initial occurrence of AP. ${ }^{1,2,4,8}$ Chronic pancreatitis (CP), in which children have imaging or functional evidence of irreversible pancreatic damage, ${ }^{9}$ is estimated to have an incidence of $\sim 2$ per 100,000 children per year. ${ }^{7,10}$ Both ARP and CP are associated with significant disease burden. ${ }^{11,12}$ Children experience frequent abdominal pain, emergency room (ER) visits, and hospitalizations. They usually undergo numerous endoscopic and surgical procedures. INSPPIRE is the first multicenter, multidisciplinary collaboration that seeks to examine pancreatitis in children. ${ }^{9,11-17}$ and to define the characteristics and sequelae associated with ARP or CP. ${ }^{18-22}$

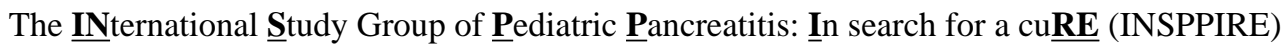
Consortium was formed to systematically characterize ARP and CP in childhood and address the knowledge gap in pediatric pancreatitis. The work done through INSPPIRE Consortium, funded by a National Institute of Diabetes and Digestive and Kidney Diseases (NIDDK), R21 grant, has led to the establishment of the first multicenter pediatric pancreatitis cohort study, the INSPPIRE cohort. ${ }^{14}$ The INSPPIRE Study has enrolled over 500 children with ARP or CP and begun to define the risk factors, natural history, disease burden, and outcome of pediatric ARP and CP. ${ }^{9,11-17,23-27}$ INSPPIRE has provided a much more detailed cross-sectional assessment of pediatric ARP and CP than was previously available, but its longitudinal contribution was limited. Data on disease progression and treatment efficacy in children has so far remained largely retrospective. Importantly, the lack of patient data and understanding of disease pathogenesis make it difficult to design and implement therapeutic trials in children. 
Multiple barriers hinder advances in our understanding of pancreatitis in children. Although $\mathrm{ARP}$ and $\mathrm{CP}$ are more common than previously estimated, they are not common enough for single-center studies to provide definitive answers. Only through a prospective, longitudinal, and multi-center approach can we address the fundamental gaps in the knowledge of pediatric ARP and CP.

In 2015, the INSPPIRE Consortium became a member of the Consortium for the Study of Chronic Pancreatitis, Diabetes and Pancreatic Cancer (CPDPC), ${ }^{28}$ funded by the NIDDK and the National Cancer Institute that led to forming the INSPPIRE 2 Cohort. In this report, we describe our efforts to build the INSPPIRE 2 cohort within CPDPC, as well as the rationale, study design, sample size, statistics. and future plans.

\section{Objectives and Hypothesis}

The primary objectives of the INSPPIRE 2 study are (1) to comprehensively characterize the pediatric population with ARP and $\mathrm{CP}$ and (2) to determine predictors for early onset $\mathrm{CP}$ and its sequelae including disease burden, exocrine pancreatic insufficiency (EPI), and diabetes. INSPPIRE 2 will provide a longitudinal cohort of well-phenotyped patients and biological samples to achieve these objectives and for future research defining the pathogenesis and testing novel therapies.

We hypothesize that childhood-onset ARP follows a severe disease course with rapid progression to $\mathrm{CP}$ and early development of complications including persistent abdominal pain, growth and nutritional disturbances, EPI, glycemic abnormalities, diabetes, repeated hospitalizations and procedures, all of which result in socioeconomic burden and impaired quality of life. We also hypothesize that specific risk factors predispose children to early progression from ARP to $\mathrm{CP}$, and specific risk factors predispose children to $\mathrm{CP}$ sequelae and high disease burden. Furthermore, the prospective collection of biological samples from children with ARP and CP will provide a framework to develop biomarkers for early diagnosis of CP in children with ARP and identify disease predictors for its complications (i.e. chronic abdominal pain, growth and nutritional disturbances, EPI, glycemic abnormalities, diabetes).

\section{INSPPIRE 2 PROTOCOL}

\section{Transition From INSPPIRE to INSPPIRE 2 Cohort}

Consortium for the Study of Chronic Pancreatitis, Diabetes, and Pancreatic Cancer is a National Institutes of Health (NIH) U01 funded consortium that aims to comprehensively characterize clinical, epidemiological, and biological aspects of patients with CP (including those with ARP) and to gain insight into the pathophysiology of CP and its sequelae. Sequelae of interest include chronic abdominal pain, EPI, type 3 diabetes mellitus (T3cDM) and pancreatic cancer, in both pediatric and adult populations. ${ }^{28}$ The pediatric aspect of CPDPC began as a Pancreatic Interest Group (PIG) in 2009, which later coalesced as the

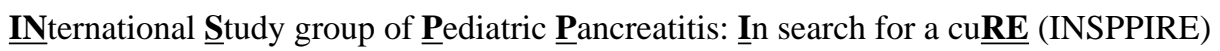
group. ${ }^{14}$ INSPPIRE started as a consortium of 14 institutions that grew to 18 , including international collaborators (Australia, Israel, and Canada). The goals of INSPPIRE were to: 
i) collect longitudinal data and DNA samples from carefully phenotyped children with ARP or CP; ii) create a network of pediatric centers to perform prospective clinical studies; iii) define risk factors that predispose children to ARP and the development of CP, EPI, and T3cDM; and iv) develop diagnostic or therapeutic guidelines for pediatric ARP and CP through prospective studies. To achieve these goals, we first developed definitions of AP, ARP, and CP and surveyed practice trends at INSPPIRE sites. ${ }^{9}$ This was followed by the formation of a prospective database and sample repository for children with ARP or $\mathrm{CP}^{14}$

In 2015, INSPPIRE joined CPDPC, and a new cohort of pediatric ARP or CP was conceptualized. This cohort, called INSPPIRE 2, was planned to build on the structure of and lessons learned from INSPPIRE, add more pediatric sites, change the governance structure, and expand to include nutritional and developmental outcomes and quality of life assessment tools to monitor pain, emotions, and behavior.

\section{INSPPIRE 2 Study Design}

INSPPIRE 2 is a prospective, observational cohort study. A total of 860 patients under 18 years of age with ARP or CP will be enrolled, including as many of the $>500$ patients from the INSPPIRE database as possible. ${ }^{14}$ Patient questionnaires and physician surveys will be applied at the time of enrollment and annually thereafter. After turning 18 years of age, patients must sign an Institutional Review Board (IRB)-approved consent form to continue the study. Data will be collected on several domains including etiology, disease course, medical interventions, and surgical interventions.

\section{INSPPIRE 2 Governance Structure and Participating Centers}

With the establishment of U01 funding, MD Anderson became the Coordinating and Data Management Center (CDMC) of the CPDPC, assuming the functions of the University of Iowa for data management, governance, and analysis. The INSPPIRE group added 4 additional sites after joining CPDPC, mainly by including the pediatric sites associated with participating adult sites. University of Iowa continues to oversee INSPPIRE 2 and houses the INSPPIRE principle investigator (PI) (A.U.), a clinical study coordinator and grant manager for study support and oversight in all 22 sites, overall research direction of the INSPPIRE 2 and subcontracting of most sites (except University of Indiana, Stanford, and Cedars-Sinai subcontracted by CDMC). The participating INSPPIRE 2 centers are listed in Table 1. INSPPIRE 2's effort requires significant logistical support. Each center obtains IRB approval locally and establishes sub-contracts with University of Iowa (18) or CDMC (3), all overseen by NIDDK. This process is complex, but ensures that the study design, data collection methods, and other study elements are robust and widely applicable.

The INSPIRRE group holds monthly calls, meets in person two times per year, and contributes to the CPDPC structure by chairing the Pediatric ARP/CP Working Group (A.U. and M.E.L.) and with members on the Steering Committee (A.U. and M.E.L.), Publication Committee (A.U., M.E.L., M.B.H., S.J.S.), Ethics Committee (S.J.S.) and Ancillary Studies Committee (A.U.). INSPPIRE projects or research ideas are first submitted by its members. Under the direction of its PI (A.U.), INSPPIRE typically forms subcommittees of 3 to 4 members to determine a study question, which is then approved and reviewed by 
INSPPIRE's Steering Committee and INSPPIRE investigators as a whole. In particular, the data interpretation, statistical analysis, and manuscript preparation are reviewed by all INSPPIRE members before a manuscript is submitted to a journal. Any organizational issues or disputes are resolved by the INSPPIRE's Executive Committee. Even during the transition between funding, cohorts and restructuring, INSPPIRE has continued to contribute productively to the literature on pediatric ARP and CP. INSPPIRE has produced several peer-reviewed publications based on the original INSPIRRE data and has given numerous presentations nationally and internationally.

\section{Inclusion/Exclusion Criteria}

Children that meet the diagnostic criteria for ARP or CP can be enrolled into the study. Acute recurrent pancreatitis is defined as at least 2 episodes of acute pancreatitis with complete resolution of pain and $\geq 1$ month pain-free interval in between episodes. ${ }^{9}$ Chronic pancreatitis inclusion criteria are listed in Table 2. Children who have significant medical illnesses that in the investigator's opinion cannot be adequately controlled with appropriate therapy or would compromise the patient's ability to tolerate study interventions are excluded from the study. All subjects who meet the eligibility criteria, and for whom parental consent and child assent is obtained, are registered with the CPDPC's CDMC prior to initiating study interventions. After the subject is registered by the participating INSPPIRE 2 site to the CDMC's Integrated Information Management System (IIMS) database, data are entered. All parents must sign an informed consent and older children should give an assent indicating that they are aware of the investigational nature of this study. All data is collected in a de-identified fashion. Since the approval of the CPDPC INSPPIRE 2 protocol in 2017, INSPPIRE 2 has enrolled 211 patients into the IIMS database.

\section{Study Procedures at Baseline and Follow-Up}

INSPPIRE 2 Study procedures are summarized in Table 3. Briefly, patients or parents and physicians will complete a form very similar to the form used in the original INSPPIRE protocol, ${ }^{9}$ detailing information on demographics, past medical history, family and social history, medications, hospitalizations, risk factors, diagnostic evaluation, treatments, outcome, and health-related quality of life. Information collected on paper questionnaires will then be transferred to the IIMS database, tabulated, and analyzed.

\section{Disease Burden}

Wherever possible, INSPPIRE 2 will utilize validated, age-appropriate assessment tools and definitions for key domains, including:

Pain-The pattern of pain (constant versus episodic), its frequency, duration, visits to the emergency room (ER) or hospitalizations for pain, and impact of pain on quality of life will be recorded in both patient and provider questionnaires. Pain intensity will be measured at enrollment, during an attack and annually using Faces Pain Scale-Revised, a self-report questionnaire validated for children $\geq 4$ years-old. ${ }^{29}$ The names, dosing, and frequency of medications taken for pain will also be queried. 
Health-Related Quality of Life-Age-specific instruments, validated for U.S. children, will be used to measure health-related quality of life (HRQoL) at enrollment and annually thereafter. These questionnaires capture physical functioning, social, emotional, physical and behavioral limitations, bodily pain, general behavior, mental health, self-esteem, general health perceptions, change in health, and parental emotional impact. Parents of children 518 years old will complete Child Health Questionnaire Parent Form (50 questions; CHQ PF-50).$^{30}$ Children $\geq 10$ years old will answer the Child Health Questionnaire Child Form (87 questions; CHQ-87). ${ }^{30}$ Adults $>18$ years old will not complete a health-related questionnaire.

Depression and Anxiety-Depression and anxiety are strong predictors of chronic pain and pain-related disability in children overall, ${ }^{31}$ but these relationships have not been comprehensively studied in children with ARP or CP. We will assess for depression and anxiety in the INSPPIRE 2 cohort at enrollment and annually thereafter. We will utilize the Child Behavioral Checklist (CBCL), one of the most widely-used standardized measures in child psychology for evaluating maladaptive behavioral and emotional problems in children.

${ }^{32}$ For preschool-age children, the CBCL/1/1/2-5 (completed by parents or surrogates) will be used. For school-age children 6-18 years of age, we will utilize CBCL/6-18 years old (completed by parents or surrogates). Children who are 11-18 years old will answer a selfreport questionnaire (Youth-Self Report Form, YSR/11-18). The CBCL and YSR assesses internalizing (i.e., anxious, depressive, and overcontrolled) and externalizing (i.e., aggressive, hyperactive, noncompliant, and under controlled) behaviors. Adults $>18$ years old will not complete a behavioral checklist.

\section{Disease Sequelae}

The presence of EPI and glucose intolerance/diabetes will be monitored at the time of enrollment and annually thereafter. Monitoring of disease status by reviewing clinically ordered tests will include specifically:

1. Exocrine pancreatic insufficiency: defined as the presence of abnormal fecal elastase (< $100 \mathrm{ug} / \mathrm{g}$ stool on 2 separate samples $\geq 1$ month apart).

2. Diabetes or prediabetes: Monitoring for diabetes in our cohort will include once yearly assessments with fasting blood glucose (FBG) and hemoglobin A1c (HbAlc) as per American Diabetes Association Guidelines. ${ }^{33}$ Oral glucose tolerance test (OGTT) will be considered if values are prediabetic (FBG: 100$125 \mathrm{mg} / \mathrm{dl}$ or $5.6-6.9 \mathrm{mmol} / \mathrm{L}$ or HbA1c: $5.7-6.4 \%)$. For OGTT, $1.75 \mathrm{~g} / \mathrm{kg}$ of standard glucose beverage (glucola, maximum $75 \mathrm{~g}$ ) will be consumed within 10 minutes of time 0 . Blood glucose will be assessed prior to the glucose load and at 120 minutes after time 0 . From this test, glycemic status will be defined as: (1) normal glucose tolerance (NGT, fasting glucose $<100 \mathrm{mg} / \mathrm{dL}, 2$ hour glucose $<140 \mathrm{mg} / \mathrm{dL}$ ); (2) pre-diabetic based on impaired fasting glucose (IFG, fasting glucose 100-125 mg/dL) and/or impaired glucose tolerance (IGT, 2 hour glucose $140-199 \mathrm{mg} / \mathrm{dL}$ ); or (3) diabetic (DM, fasting glucose $\geq 126 \mathrm{mg} / \mathrm{dL}$ or 2 hour glucose $\geq 200 \mathrm{mg} / \mathrm{dL}$ ). Patient will be diagnosed as diabetic and follow-up OGTT will no longer be required if random plasma glucose is $\geq 200 \mathrm{mg} / \mathrm{dL}$ or 11.1 
$\mathrm{mml} / \mathrm{L}, \mathrm{FBG} \geq 126 \mathrm{mg} / \mathrm{dl}$ or $7 \mathrm{mml} / \mathrm{L}$ or $2 \mathrm{~h}$ glucose is $\geq 200 \mathrm{mg} / \mathrm{dl}$ during OGTT, or $\mathrm{HbA} 1 \mathrm{c} \geq 6.5 \% .{ }^{33}$

3. Nutritional status including micronutrient deficiency: To determine the nutritional sequelae of ARP or CP in children, we will assess for malnutrition/ obesity (weight, height, body mass index (BMI), z scores; body fat mass and lean mass as measured by Dual-energy X-ray absorptiometry or DEXA scan), fat soluble vitamin deficiencies (serum vitamin levels for A, D, E, PT/INR for vitamin K) and bone density (DEXA scan) at enrollment and annually thereafter.

4. Progression from ARP to CP: We will identify patients within our cohort that presented with ARP episodes, normal exocrine and endocrine pancreas function and normal pancreas imaging without any signs of chronicity (ARP cohort). We will identify the development of $\mathrm{CP}$ on an annual basis as long as possible, as well as development of sequelae and disease burden as listed above.

\section{Biospecimen Collection}

Blood or saliva will be collected for DNA analysis once from each study participant, ideally at enrollment. Specimen labeling and tracking procedures and supplies are provided by CPDPC's CDMC. Samples will be shipped to the University of Iowa central repository via overnight courier. The central repository will process and aliquot the specimens, extract the genomic DNA, followed by the storage of the sample at $-80^{\circ} \mathrm{C}$. At the end of the study, data and the specimens will be transferred to the NIDDK Central Repositories (https:// repository.niddk.nih.gov/home/).

\section{Power Calculation}

INSPPIRE 2 will enroll all eligible, consenting patients across the study sites. The sample size is determined by the accrual rate and not driven by any specific hypothesis or endpoint. Over 500 patients have been enrolled in INSPPIRE. Patients enrolled in INSPPIRE will be re-enrolled in INSPPIRE 2 for data and sample collection if they still meet enrollment criteria. New patients satisfying the eligibility criteria will also be enrolled into INSPPIRE 2. We expect that the cohort will reach 860 patients over the next 2 years. Given 860 patients, the $95 \%$ confidence interval for the frequency estimate of an event will not be wider than 0.067 . Given a significance level of 0.05 , we will have $80 \%$ power to detect an effect size of 0.096 between the mean of a variable at two different time points. To compare the frequency of a binary endpoint (e.g., whether ARP is progressed to CP) between groups defined by a certain baseline characteristic, assuming a significance level of 0.05 and that patients are equally divided into two groups according to the baseline characteristic, we have $80 \%$ power to detect an odds ratio of $1.81,1.60,1.52,1.49$, and 1.49 when the frequency in one group is $0.1,0.2,0.3,0.4$, and 0.5, respectively. For time-to-event endpoints (e.g., the time from ARP to $\mathrm{CP}$ ), assuming a significance level of 0.05 and that patients are equally divided into two groups according to a certain baseline characteristic, we have $84 \%$ power to detect a hazard ratio of 1.35 using the log-rank test. 


\section{Statistical Analysis Plan}

Summary statistics and graphs will be used to characterize the pediatric population, disease course and development of complications. We will compare children who have progressed to $\mathrm{CP}$ to those with ARP that have not progressed to $\mathrm{CP}$ in demographic data (age at diagnosis for AP, ARP and CP, race, sex, ethnicity); clinical data (weight, height, BMI, z scores, body fat mass, lean mass); genetic, obstructive, environmental, autoimmune risk factors; laboratory data (fold amylase and lipase elevation with each episode, hypercalcemia, hypertriglyceridemia); family history (acute and chronic pancreatitis, pancreatic cancer); emotional and behavioral issues (CBCL/YSR) in our ARP and CP cohort at enrollment and annually thereafter. Two-sample $t$-tests or Wilcoxon rank-sum tests will be used for comparing the continuous/ordinal variables and Pearson Chi-square tests for the categorical variables. The variables that suggest differences between the two groups $(P<0.05)$ will be included as independent variables in a multivariable logistic regression analysis to identify the risk factors that predispose children for early progression from ARP to CP. Cox proportional hazard regression model will further be used to assess the effect of these variables on the time to progression of CP. A similar approach will be adopted to identify the risk factors that predispose children to $\mathrm{CP}$ sequelae and high disease burden.

\section{DISCUSSION}

The INSPPIRE Consortium was founded in 2009 as a multi-center collaborative dedicated to studying the pathogenesis of pediatric ARP and CP and developing novel diagnostic and therapeutic approaches. The NIDDK R21-funded INSPPIRE Cohort developed as the product of that collaboration created the first well-phenotyped, multi-center cohort of children with ARP or CP. INSPPIRE led to several key observations, including the importance of genetic risk factors, the high disease burden in pediatric ARP and CP, and the influence of specific genetic mutations, anatomical variants, autoimmunity and age of onset on disease behavior. ${ }^{11-13,15,17,25,26}$ The INSPPIRE 2 study is an extension and expansion of that work, now under the umbrella of the NIH U01 funded CPDPC. ${ }^{28}$ Both the goals and the structure of INSPPIRE 2 parallel those of the CPDPC, with the focus on building large prospective cohorts of patients with ARP and $\mathrm{CP}$ to enable studies of etiology, progression, and treatment.

The INSPPIRE 2 Study builds on strengths from INSPPIRE. In INSPPIRE 2's first year, our 22 centers have already enrolled over 200 children into this $2^{\text {nd }}$ prospective cohort. Once fully implemented, INSPPIRE 2 will be almost twice the size of the INSPPIRE cohort, increasing power to analyze details and diversity in this heterogenous disease. Our broad geographic reach will enable us to study differences in ARP and CP by demographics, region, disease etiology, prescribed medications, previous treatments, and other factors that vary across centers. The database will be increasingly valuable as we collect more data prospectively and follow the cohort into adulthood.

INSPPIRE 2's novel aspects will significantly expand our consortium's ability to define, understand, and manage pediatric ARP and $\mathrm{CP}$. We have a broadened approach to genetic analysis of all INSPPIRE 2 samples and will collect DNA samples from all patients enrolled into the study. The biosample repository developed at the University of Iowa will provide a 
new and incredibly valuable resource, particularly for translational work on genetic contributors, biomarkers of disease progression, and innovative treatment options. Finally, the INSPPIRE 2 cohort will provide opportunities for treatment trials. Ancillary studies are planned for diagnostic and treatment approaches for pediatric ARP and $\mathrm{CP}$ as part of the CPDPC effort.

On an ongoing basis, our work within the CPDPC highlights similarities and differences between pediatric and adult pancreatitis, its causes, complications, and implications for health. For example, alcohol and smoking have long been recognized as major risk factors for $\mathrm{CP}$ in adults, ${ }^{34,35}$ but they are extremely uncommon in the pediatric age group. The current literature suggests that children with ARP or CP often have genetic variants-in particular in CFTR, SPINK1, PRSS1, and CPA1.,19,36-39 Work within the consortium has created opportunities for both pediatricians and internists on collaborative projects such as diagnostic imaging studies and pain therapies for CP. Although most children with ARP or $\mathrm{CP}$ will become adults with ARP or $\mathrm{CP}$, their risk factors and opportunities to prevent disease progression are expected to be different and environmental factors will probably influence outcomes later in life. The transition of pediatric patients to the adult cohort is an ongoing focus of the CPDPC. INSPPIRE 2's representation within CPDPC highlights the importance of research and resources that are specific to children and in their transition to adulthood.

A major strength of the INSPPIRE 2 consortium continues to be the broad participation and dedication of its investigators, coordinators, and other study personnel. This organizational structure has allowed INSPPIRE to balance efficiency and opportunities for broad participation. The infrastructure provided by the CPDPC and its CDMC has further standardized INSPPIRE 2's data collection and recording methods which will contribute to the completeness and validity of the collected data.

INSPPIRE 2's long term plan is to enroll children with ARP or CP and follow them longitudinally to determine the predictors for early onset $\mathrm{CP}$ and its sequelae including disease burden, EPI and diabetes. One ongoing aim is the analysis of the data collected, with a focus on correlating clinical information with biospecimen analyses. Ancillary studies will evaluate diagnostic and treatment approaches on this unique cohort. INSPPIRE 2 is in the beginning stages of establishing HRQoL scales for pediatric patients with ARP or CP to determine disease burden and monitor response to potential future therapies. Additionally, our long-term goal of collecting urine, and stool specimens from this patient population will aid in studies to determine early biomarkers for $\mathrm{CP}$ and its sequelae.

In summary, through the INSPPIRE 2 study, we will build a large, well-characterized, diverse prospective cohort of children with ARP or CP. The size and scope of this cohort will provide data to significantly deepen our understanding of the pathogenesis and progression for these diseases and a framework for studying interventions to slow or reverse disease progression. 


\section{ACKNOWLEDGMENTS}

The authors would like to acknowledge all the members participating in the Consortium for the study of Chronic Pancreatitis, Diabetes and Pancreatic Cancer (CPDPC):

A. Investigators and Coordinators for Adult Pancreas studies (PROCEED, DETECT, NOD studies)

1) Co-investigators, Collaborators:

Baylor College of Medicine: Mohamed O. Othman; Mandeep Bajaj; Carlos A. Farinas; George Van Buren, II; Joseph Petrosino; Eric J Silberfein; Barbara W. Trautner

Cedars Sinai Medical Center: Marc T. Goodman; Christie Y. Jeon; Srinivas Gaddam; Maxim S. Petrov; Joseph Pisegna; Sofiya Reicher; Bechien U. Wu; Laith Jamil; Simon K. Lo; Michael Stanley Lewis

Indiana University: Kieren J. Mather; Temel Tirkes; Stuart Sherman; Murray Korc

Kaiser Foundation: Margaret A. Tempero; Suraj Gupta; Kareem Mawad; Craig A, Munroe; Terry L. Jue; Assiamira Ferrara; Scott Oakes; Mark Anderson; Mattias Hebrok; Charles Quesenberry

Mayo Clinic: Yogish Kudva; Naoki Takahashi; Sudhakar Venkatesh; Gloria M. Petersen; Aleksey Matveyenko; Ayush Sharma; Tonya Palermo; Timothy B. Gardner; Yu-Xiao Yang

Ohio State University: David Bradley; Zobeida Cruz-Monserrate; Amy Hosmer; Luis Lara; Gregory Lesinski; Somashaker Krishna; Thomas Mace; Sean McCarthy; Zarine Shah; Sebastian Strobel; Jon Walker

Stanford University: Marina Basina; Bryant Lin; Shreyas Vasanawala.

University of Florida: Joseph R. Grajo; Patricia P. Moser; Amer S. Abouhamze

University of Texas MD Anderson Cancer Center: Liang Li; Ying Yuan

University of Pittsburgh: Adam Slivka; Georgios I. Papachristou; Randall E. Brand; Anil Dasyam; Jennifer Chennat; Kevin McGrath; Gong Tang; Anna E. Phillips; Aatur Singhi.

2) Coordinators and Research Associates:

Baylor College of Medicine: Delrose A. Jones; Amy McElhany; Jose E. Mendez-Reyes; Martha Navarro Cagigas; Linda H. Nguyen

Cedars-Sinai Medical Center: Cindy Miao; Phoebe Chang; Yunhee Choi-Kuaea

Indiana University: Sheryl Lynch; Vanessa Patrick; Alicia Winters; Gail McNulty; Suzette Schmidt

Kaiser Foundation: Amethyst D. Leimpeter; Erica H. Kerezsi; Jun Shan; Desiree Goldstein; Keeli McClearnen

Mayo Clinic: Vincent Anani; Natasha Kanwar

Ohio State University: Emily Bowns; Alejandra Cervantes; Brianna Conley; Casey McClurkin

Stanford University: Judith Chuang; Christina Vu; Yujun Yang; Sangbin Park

University of Florida: Amber Bouton; April A. Goddard; Danielle Poulton

University of Texas MD Anderson Cancer Center: Ria Ghosh; Noemy Contreras; Lavinia Padmore; Savitri Appana

University of Pittsburgh: Laura Mathews; Kelley Woods; Kimberly Stello; Nicole Komara; Melissa Saul; Melanie Mays; Phil Greer; Juan Castaneda; Amanda Kirshkaln; Mark Batistick

B. INSPPIRE 2 Coordinators

Children's Hospital Los Angeles: Roy Leong 
Children's Hospital of Philadelphia: Lucia Sanchez

Cincinnati Children's Hospital: Tyler Thompson

Hadassah University Hospital: Batya Vertsman; Geulah Cramel-Fink

Massachusetts General Hospital: Ana Fernandes; Francine Molay

Medical College of Wisconsin; Adriane Mueller; Sydnee Rausch

Nationwide Children's Hospital: Ling Fan; Mohammad Ovais

Primary Children's Hospital: Cameron Tear; Cynthia Jones; Richard Drake

Seattle Children's Hospital: Stephanie Lammers; Kara Cooper

Sydney Children's Hospital: Sandra Lowe

University of California, San Francisco: Melissa Zerofsky

University of Iowa: Gretchen Cress; Karen Scudiero

University of Minnesota: Cathy Kneeland

University of Pittsburgh: Kathleen Calabro; Adam Kufen

University of Texas Southwestern Medical Center: Amber Crabb; Lauren Plummer

Washington University: Ashley Simpson

Funding: Research reported in this publication was supported by National Cancer Institute and National Institute of Diabetes and Digestive and Kidney Diseases of the National Institutes of Health under award numbers R21DK096327, R01DK097820, U01DK108334, U01DK108300, U01DK108327, U01DK108326, U01DK108323, U01DK108328, U01DK108314, U01DK108300. The content is solely the responsibility of the authors and does not necessarily represent the official views of the National Institutes of Health.

\section{REFERENCES}

1. Lopez MJ. The changing incidence of acute pancreatitis in children: a single-institution perspective. J Pediatr. 2002;140:622-624. [PubMed: 12032533]

2. Werlin SL, Kugathasan S, Frautschy BC. Pancreatitis in children. J Pediatr Gastroenterol Nutr. 2003;37:591-595. [PubMed: 14581803]

3. Nydegger A, Heine RG, Ranuh R, et al. Changing incidence of acute pancreatitis: 10-year experience at the Royal Children's Hospital, Melbourne. J Gastroenterol Hepatol. 2007;22:13131316. [PubMed: 17489962]

4. Sánchez-Ramírez CA, Larrosa-Haro A, Flores-Martínez S, et al. Acute and recurrent pancreatitis in children: etiological factors. Acta Paediatr. 2007;96:534-537. [PubMed: 17306005]

5. Park A, Latif SU, Shah AU, et al. Changing referral trends of acute pancreatitis in children: A 12year single-center analysis. J Pediatr Gastroenterol Nutr. 2009;49:316-322. [PubMed: 19503003]

6. Morinville VD, Barmada MM, Lowe ME. Increasing incidence of acute pancreatitis at an American pediatric tertiary care center: is greater awareness among physicians responsible? Pancreas. 2010;39:5-8. [PubMed: 19752770]

7. Sellers ZM, MacIsaac D, Yu H, et al. Nationwide Trends in Acute and Chronic Pancreatitis Among Privately Insured Children and Non-elderly Adults in the United States, 2007-2014. Gastroenterology. 2018;155:469-478. e1. [PubMed: 29660323]

8. Pezzilli R, Morselli-Labate AM, Castellano E, et al. Acute pancreatitis in children. An Italian multicentre study. Dig.Liver Dis. 2002;34:343-348. [PubMed: 12118952]

9. Morinville VD, Husain SZ, Bai H, et al. Definitions of pediatric pancreatitis and survey of present clinical practices. J Pediatr Gastroenterol Nutr. 2012;55:261-265. [PubMed: 22357117] 
10. Pant C, Sferra TJ. Emergency Department Visits and Hospitalizations in Children With Chronic Pancreatitis in the United States. J Pediatr Gastroenterol Nutr. 2015;61:568-570. [PubMed: 26111296]

11. Kumar S, Ooi CY, Werlin S, et al. Risk Factors Associated With Pediatric Acute Recurrent and Chronic Pancreatitis: Lessons From INSPPIRE. JAMA Pediatr. 2016;170:562-569. [PubMed: 27064572]

12. Ting J, Wilson L, Schwarzenberg SJ, et al. Direct Costs of Acute Recurrent and Chronic Pancreatitis in Children in the INSPPIRE Registry. J Pediatr Gastroenterol Nutr. 2016;62:443-449. [PubMed: 26704866]

13. Husain SZ, Morinville V, Pohl J, et al. Toxic-metabolic Risk Factors in Pediatric Pancreatitis: Recommendations for Diagnosis, Management, and Future Research. J Pediatr Gastroenterol Nutr. 2016;62:609-617. [PubMed: 26594832]

14. Morinville VD, Lowe ME, Ahuja M, et al. Design and implementation of INSPPIRE. J Pediatr Gastroenterol Nutr. 2014;59:360-364. [PubMed: 24824361]

15. Schwarzenberg SJ, Bellin M, Husain SZ, et al. Pediatric chronic pancreatitis is associated with genetic risk factors and substantial disease burden. J Pediatr. 2015;166:890-896.e1. [PubMed: 25556020]

16. Scheers I, Palermo JJ, Freedman S, et al. Recommendations for Diagnosis and Management of Autoimmune Pancreatitis in Childhood: Consensus from INSPPIRE. J Pediatr Gastroenterol Nutr. J Pediatr Gastroenterol Nutr. 2018;67:232-236. [PubMed: 29746340]

17. Lin TK, Abu-El-Haija M, Nathan JD, et al. Pancreas Divisum in Pediatric Acute Recurrent and Chronic Pancreatitis: Report From INSPPIRE. J Clin Gastroenterol. 622018 [Epub ahead of print].

18. Wang W, Liao Z, Li ZS, et al. Chronic pancreatitis in Chinese children: etiology, clinical presentation and imaging diagnosis. J Gastroenterol.Hepatol. 2009;24:1862-1868. [PubMed: 19793170]

19. Lucidi V, Alghisi F, Dall'Oglio L, et al. The etiology of acute recurrent pancreatitis in children: a challenge for pediatricians. Pancreas. 2011;40:517-521. [PubMed: 21499205]

20. Sultan M, Werlin S, Venkatasubramani N. Genetic prevalence and characteristics in children with recurrent pancreatitis. J Pediatr Gastroenterol Nutr. 2012;54:645-650. [PubMed: 22094894]

21. Minen F, De Cunto A, Martelossi S, et al. Acute and recurrent pancreatitis in children: exploring etiological factors. Scand J Gastroenterol. 2012;47:1501-1504. [PubMed: 23016884]

22. Chowdhury SD, Chacko A, Ramakrishna BS, et al. Chronic Pancreatitis In Children. Indian Pediatr. 2013;50:1016-1019. [PubMed: 23798627]

23. Pohl J, Morinville V, Husain SZ, et al. Toxic-Metabolic Risk Factors Are Uncommon in Pediatric Chronic Pancreatitis. J Pediatr Gastroenterol Nutr. 2016;62:e66-e67.

24. Gariepy CE, Heyman MB, Lowe ME, et al. Causal Evaluation of Acute Recurrent and Chronic Pancreatitis in Children: Consensus From the INSPPIRE Group. J Pediatr Gastroenterol Nutr. 2017;64:95-103. [PubMed: 27782962]

25. Giefer MJ, Lowe ME, Werlin SL, et al. Early-Onset Acute Recurrent and Chronic Pancreatitis Is Associated with PRSS1 or CTRC Gene Mutations. J Pediatr. 2017;186:95-100. [PubMed: 28502372]

26. Scheers I, Palermo JJ, Freedman S, et al. Autoimmune Pancreatitis in Children: Characteristic Features, Diagnosis, and Management. Am J Gastroenterol. 2017;112:1604-1611. [PubMed: 28374818]

27. Troendle DM, Fishman DS, Barth BA, et al. Therapeutic Endoscopic Retrograde Cholangiopancreatography in Pediatric Patients With Acute Recurrent and Chronic Pancreatitis: Data From the INSPPIRE (INternational Study group of Pediatric Pancreatitis: In search for a cuRE) Study. Pancreas. 2017;46:764-769. [PubMed: 28609364]

28. Serrano J, Andersen DK, Forsmark CE, et al. Consortium for the Study of Chronic Pancreatitis, Diabetes and Pancreatic Cancer: From Concept to Reality. Pancreas. 2018;47:\#\#\#-\#\#\#\#.

29. Hicks CL, von Baeyer CL, Spafford PA, et al. The Faces Pain Scale-Revised: toward a common metric in pediatric pain measurement. Pain. 2001;93:173-183. [PubMed: 11427329] 
30. Landgraf JM, Abetz L, Ware J. The CHQ User's Manual. 1st Edition ed. Boston MA: Health Institute at New England Medical Center; 1996.

31. Ericsson M, Poston WS, Linder J, et al. Depression predicts disability in long-term chronic pain patients. Disabil Rehabil. 2002;24:334-340. [PubMed: 12017467]

32. Achenbach TM, Ruffle TM. The Child Behavior Checklist and related forms for assessing behavioral/emotional problems and competencies. Pediatr Rev. 2000;21:265-271. [PubMed: 10922023]

33. 2. Classification and Diagnosis of Diabetes: Standards of Medical Care in Diabetes-2018. Diabetes Care. 2018;41(Suppl 1):S13-S27. [PubMed: 29222373]

34. Mayerle J, Hoffmeister A, Werner J, et al. Chronic pancreatitis--definition, etiology, investigation and treatment. Dtsch Arztebl Int. 2013;110:387-393. [PubMed: 23826027]

35. Beer S, Zhou J, Szabo A, et al. Comprehensive functional analysis of chymotrypsin C (CTRC) variants reveals distinct loss-of-function mechanisms associated with pancreatitis risk. Gut. 2013;62:1616-1624. [PubMed: 22942235]

36. Schnur A, Beer S, Witt H, et al. Functional effects of 13 rare PRSS1 variants presumed to cause chronic pancreatitis. Gut. 2014;63:337-343. [PubMed: 23455445]

37. van Geenen EJ, Smits MM, Schreuder TC, et al. Smoking is related to pancreatic fibrosis in humans. Am J Gastroenterol. 2011;106:1161-1166; quiz 1167. [PubMed: 21577244]

38. Witt H Gene mutations in children with chronic pancreatitis. Pancreatology. 2001;1:432-438. [PubMed: 12120220]

39. Witt H, Beer S, Rosendahl J, et al. Variants in CPA1 are strongly associated with early onset chronic pancreatitis. Nat Genet. 2013;45:1216-1220. [PubMed: 23955596] 
Table 1.

\title{
INSPPIRE Centers
}

\author{
University of Iowa, Iowa City, Iowa (main site) \\ Children's Hospital of Pittsburgh, Pittsburgh, Penn \\ Baylor College of Medicine, Houston, Texas \\ University of Minnesota, Minneapolis, Minn \\ University of Texas Southwestern Medical School (UTSW), Dallas, Texas \\ Medical College of Wisconsin (MCW), Milwaukee, Wis \\ University of California, San Francisco (UCSF), San Francisco, Calif \\ Nationwide Children's Hospital, Columbus, Ohio \\ University of Utah, Salt Lake City, \\ University of Washington in Seattle, Seattle, Wash \\ McGill University, Montreal, Canada \\ University of Toronto, Toronto, Canada \\ University of New South Wales (UNSW), Sydney, Australia \\ Hadassah University Hospitals, Jerusalem, Israel \\ Cincinnati Children's Hospital Medical Center, Cincinnati, Ohio \\ Children's Hospital of Los Angeles, Los Angeles, Calif \\ Children's Hospital of Philadelphia, Philadelphia, Penn \\ Massachusetts General Hospital Children's, Boston, Mass \\ Washington University in St. Louis, St. Louis, Mo \\ Stanford University, Stanford, Calif \\ Indiana University, Indianapolis, Ind \\ Cedars-Sinai Medical Center, Los Angeles, Calif
}


Table 2.

\section{Definitions of Pediatric CP}

Children with irreversible structural changes* in the pancreas with or without abdominal pain; OR with exocrine pancreatic insufficiency; OR with diabetes will be classified as CP.

Definition of irreversible structural changes:

- *Ductal calculi, dilated side branches, parenchymal calcifications found in any imaging (abdominal ultrasound, magnetic resonance imaging/magnetic resonance cholangiopancreatography (MRI/MRCP), computerized tomography (CT), endoscopic retrograde cholangiopancreatography (ERCP), endoscopic ultrasound (EUS).

- Ductal obstruction or stricture/dilatation/irregularities that are persistent (for $>2$ months) on any imaging.

- Parenchymal atrophy, irregular contour, accentuated lobular architecture, cavities alone are not diagnostic findings for CP.

- Surgical or pancreatic biopsy specimen demonstrating histopathologic features compatible with CP (acinar atrophy, fibrosis, protein plugs, infiltration with lymphocytes, plasma cells, macrophages). 
Table 3.

Study Procedures

\begin{tabular}{lc}
\hline & Baseline or Follow up \\
\hline Physician questionnaire-Baseline or Follow-up form & $\mathrm{X}$ \\
Patient questionnaire-Baseline or Follow-up form & $\mathrm{X}$ \\
CHQ-Child/Parent & $\mathrm{X}$ \\
CBCL Form-Child/Parent & $\mathrm{X}$ \\
DNA sample-only at baseline & $\mathrm{X}$ \\
Weight, height, body mass index & $\mathrm{X}$ \\
Fecal elastase (if available and performed as a standard of care test) & $\mathrm{X}$ \\
Amylase, lipase elevation with each AP, hypercalcemia, hypertriglyceridemia (if available and performed as a & $\mathrm{X}$ \\
standard of care test) & $\mathrm{X}$ \\
Fasting glucose/HbA1c (if available and performed as a standard of care test) & $\mathrm{X}$ \\
OGTT or MMTT (if available and performed as a standard of care test) & $\mathrm{X}$ \\
Vitamins A, D, E (if available and performed as a standard of care test) & $\mathrm{X}$ \\
DEXA scan (if available and performed as a standard of care test) & $\mathrm{X}$ \\
Imaging Studies (if available and performed as a standard of care test) - (CT, MRCP, EUS, ERCP, US, etc) & \\
CHQ indicates Child Health Questionnaires; CBCL, Childhood Behavior Checklist & \\
\hline
\end{tabular}

